\title{
Aplicación de herramientas de clase mundial para la gestión de mantenimiento en empresas cementeras basado en la metodología $\mathrm{MCC}$
}

\section{Application of world-class tools for managing maintenance in cement companies based on the methodology MCC}

\section{Aplicação de ferramentas de classe mundial para a gestão de manutenção em fabricas de cimento baseado na metodologia da MCC}

\author{
Jessica Yajaira Uzcátegui-Gutiérrez ${ }^{1}$, Andrónico Varela-Cárdenas² ${ }^{2}$ Juan Isidro Díaz-García ${ }^{3}$
}

Forma de citar: J. Y. Uzcátegui-Gutiérrez, A. Varela-Cárdenas, J. I. Díaz-García, “Aplicación de herramientas de clase mundial para la gestión de mantenimiento en empresas cementeras basado en la metodología MCC", Respuestas, vol. 21, no. 1, pp. 77-88, 2016.

Recibido:

Abril 14 de 2015

Aceptado:

Septiembre 22

de 2015

${ }^{1}$ Magíster en Mantenimiento Industrial

jessica.uzcategui@unet.edu.ve Orcid: 0000-0002-3496-2879

Universidad Nacional

Experimental del Táchira

San Cristóbal - Venezuela

${ }^{2}$ Magíster en Ingeniería Industrial

avarelac@unet.edu.ve Orcid: 0000-0001-8823-7739

Universidad Nacional

Experimental del Táchira

San Cristóbal - Venezuela

${ }^{3}$ Magíster en Ciencia de los Materiales

jidiaz@unet.edu.ve Orcid: 0000-0002-2650-793X

Universidad Experimental del Táchira

San Cristóbal - Venezuela

\section{Resumen}

Antecedentes: La categoría de clase mundial en mantenimiento se apoya en metodologías como el Mantenimiento Centrado en Confiabilidad (MCC), la cual ayuda a determinar acciones concretas de mantenimiento, identificando fallas y procedimientos de corrección de las mismas. Objetivos: En este trabajo se planteó el diseño de un marco referencial para la aplicación de herramientas para la gestión mantenimiento de empresas cementeras, aplicando la metodología del MCC. Método: Se desarrolló una investigación de tipo descriptiva y de campo, en la cual se consideró como unidad de análisis la Fábrica Nacional de Cemento, Planta Táchira en Venezuela. A tal efecto, la técnica de recolección de datos constató indicios escritos y el instrumento seleccionado fue una matriz de categorías. Resultado: Se diagnosticó que la gestión de mantenimiento en la empresa no considera los principios básicos de la confiabilidad de los activos. Conclusiones: Se identificaron las etapas de aplicación de las herramientas de confiabilidad: análisis de criticidad, análisis de modos y efectos de fallas, y análisis de causa - raíz, a través del cumplimiento de tres fases contentivas en un sistema que involucra insumos, procesos y resultados en cada fase; para finalmente indicar las pautas a seguir en la aplicación de las mencionadas herramientas.

Palabras Clave: cementera, confiabilidad, herramientas del MCC, Mantenimiento.
Abstract
Background: The category of world class maintenance is based on methodologies such as Reliability Centered Maintenance, which helps to determine specific maintenance actions, identifying fails and the procedures for fixing them. Objectives: In this work the design of a framework was proposed to implement management tools for maintenance of ce- ment companies, applying the methodology of the MCC. Method: A descriptive and field research was developed, in which it was considered as a unit of analysis the National Ce- ment Factory, Plant Tachira in Venezuela. To do this, the data collection technique verified written evidence and an array of categories was the selected instrument. Result: It was diagnosed that the maintenance management in the company does not consider the basic principles of asset reliability. Conclusions: The stages of application of the tools of reli- ability were identified: criticality analysis, analysis of failure modes and effects, and root 
No. 1

Enero - Junio 2016 ISSN 0122-820X E-ISSN 2422-5053 cause analysis, through compliance three contentive phases in a system involving inputs, processes and results in each phase; to finally indicate the guidelines in the application of the above-mentioned tools.

Keywords: cement, reliability, tools MCC, maintenance.

\section{Resumo}

Antecedentes: A categoria de classe mundial em manutenção se apoia na metodologia de Manutenção Centrada em Confiabilidade (MCC), que ajuda a determinar ações concretas de manutenção, identificando falhas e procedimentos de autocorreção. Objetivos: Neste trabalho foi proposta uma guia de referência para a aplicação de ferramentas de gestão de manutenção para fabricas de cimento, aplicando a metodologia da MCC. Método: Desenvolveu-se uma pesquisa de tipo descritiva e de campo, na qual se considerou como unidade de análise a Fábrica Nacional de Cimento, Planta Táchira na Venezuela. Para esta finalidade, a técnica de recolecção de dados conseguiu constatar indícios escritos e o instrumento selecionado foi una matriz de categorias. Resultados: Diagnosticou-se que a gestão de manutenção na fábrica de cimento não considerava os princípios básicos da confiabilidade dos ativos. Conclusões: Identificaram-se as etapas de aplicação das ferramentas de confiabilidade: análise de criticidade, análise de modos e efeitos de falhas, e análise de causa - raiz, através do cumprimento de três fases contentivas num sistema que involucra insumos, processos e resultados em cada fase; para finalmente indicar as orientações a seguir na aplicação das mencionadas ferramentas.

Palavras-chaves: confiabilidade, fábrica de cimento, ferramentas da $\mathrm{MCC}$, manutenção

\section{Introducción}

Las demandas actuales en el sector productivo del cemento, requieren procesos eficientes, seguros, confiables y altamente competitivos, que lleven la producción nacional a niveles superiores con el uso óptimo de los recursos disponibles. Trabajos anteriores, como el desarrollado por la consultora venezolana Main Tech: RCM en Plantas Cementeras [1], o el patrocinado por el Centro de Excelencia de Producción de Petróleos de Venezuela S.A.: Modelo Integral para Optimizar la Fiabilidad en Instalaciones Petroleras [2], dentro del Programa Corporativo de Gerencia Integral de Activos, han marcado una iniciativa para la implantación de metodologías de clase mundial en la búsqueda de elevar estándares de desempeño y producción, de bienes y servicios en el país. A tal efecto, el Mantenimiento Centrado en Confiabilidad [3], permite identificar acciones de mantenimiento más costo - efectivas que se ajusten a los patrones de falla de los equipos, a través del análisis de estado real de los mismos [4]-[5]. En el trabajo que se presenta, fueron resueltas las interrogantes: ¿Puede diseñarse un marco referencial para aplicar herramientas de clase mundial? ¿En las empresas cementeras la gestión de mantenimiento considera aspectos relacionados con la confiabilidad de los activos? ¿Cómo se definen las herramientas basadas en la metodología del MCC, para mejorar la gestión de mantenimiento? Para lo anterior, se desarrolló una investigación atendiendo como unidad de análisis la Fábrica Nacional de Cemento, Planta Táchira en Venezuela, donde la vinculación de las acciones rutinarias con metodologías de clase mundial [6], permitirá finalmente satisfacer la creciente necesidad de tener una alta disponibilidad en los sistemas productivos de cemento, dada la relación de este sector con el desarrollo productivo del país. 


\section{Materiales y métodos}

En cuanto a materiales fueron utilizados: Norma SAE JA 1011 [7], JA 1012 [8], papelería, equipos de computación, grabadora y bibliografía actualizada en el tema.

En cuanto a los métodos, se empleó:

- Entrevistas no estructuradas al personal del departamento de mantenimiento de la Fábrica Nacional de Cemento Planta Táchira donde se describió la naturaleza del problema.

- Revisión documental de las normas internacionales vigentes en cuanto a los términos y aplicación del Mantenimiento Centrado en Confiabilidad, para la elaboración de la Matriz de Categorías como instrumento de recolección de datos.

- Auditoría del sistema de gestión de mantenimiento en la fábrica en aspectos inherentes a la confiabilidad de los equipos sujetos a mantenimiento del proceso productivo.

- Análisis cualitativo y cuantitativo de la data recolectada en el diagnóstico de la gestión de mantenimiento.

- Diseño de fases del marco de aplicación de las herramientas de confiabilidad en función de las oportunidades de mejora identificadas en el diagnóstico.

\section{Resultados y discusión}

Una vez analizada la norma SAE JA1012, y considerando también el contexto operacional de la empresa, se aplicó el instrumento de recolección de datos, el cual con la forma de auditoría, evaluó el grado de cumplimiento de la gestión actual de mantenimiento con la mencionada norma, a través de siete capítulos. El resultado se detalla en la tabla I, en la cual puede apreciarse la puntuación total de la evaluación el cual fue de 225 sobre 1700 puntos.

Tabla I. Resultado de la Evaluación Global con la Matriz de Categorías en la Fábrica Nacional de Cemento, Planta Táchira

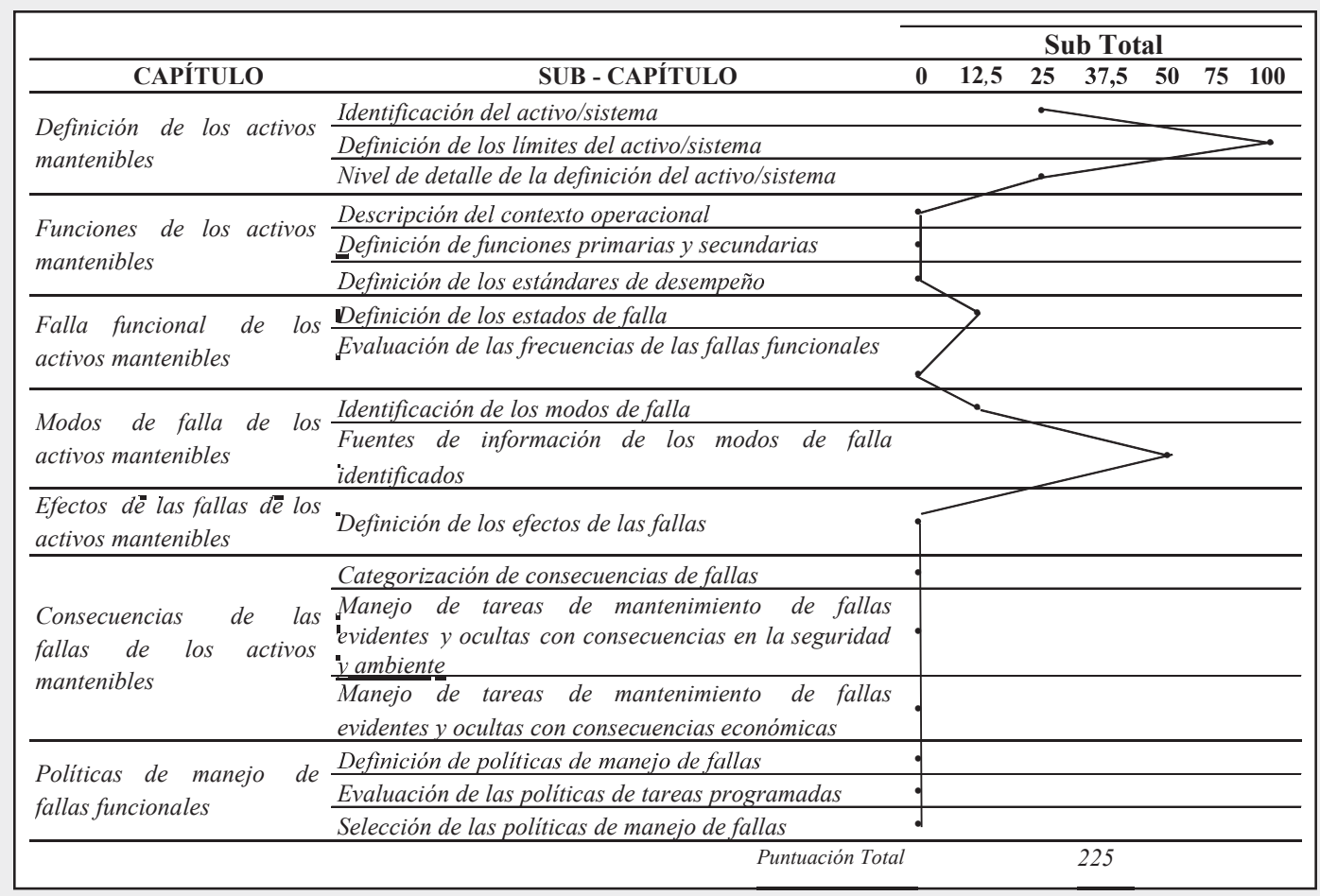


No. 1

Enero - Junio 2016 ISSN 0122-820X E-ISSN 2422-5053
De acuerdo con los resultados del diagnóstico y en función de los niveles establecidos en la tabla II, la gestión de mantenimiento en la empresa Fábrica Nacional de Cemento, Planta Táchira, no considera los principios básicos de la confiabilidad de los activos.

Tabla II. Nivel de cumplimiento de una gestión de mantenimiento con la confiabilidad de activos de una empresa cementera en función de la puntuación total obtenido en la matriz de categorías

\begin{tabular}{|c|c|}
\hline & NIVEL DE CUMPLIMIENTO DE UNA GESTIÓN DE \\
\hline $\begin{array}{l}\text { PUNTUACION } \\
\text { TOTAL }\end{array}$ & $\begin{array}{l}\text { MANTENIMIENTO, CON LA CONFIABILIDAD DE ACTIVOS DE } \\
\text { UNA EMPRESA CEMENTERA }\end{array}$ \\
\hline $0-400$ & $\begin{array}{l}\text { La gestión de mantenimiento en la empresa no considera los principios } \\
\text { básicos de la confiabilidad de activos }\end{array}$ \\
\hline $400-800$ & $\begin{array}{l}\text { La gestión de mantenimiento en la empresa considera algunos de los princi- } \\
\text { pios básicos de la confiabilidad de activos }\end{array}$ \\
\hline $800-1200$ & $\begin{array}{l}\text { La gestión de mantenimiento en la empresa está orientada hacia los princi- } \\
\text { pios básicos de la confiabilidad de activos }\end{array}$ \\
\hline $1200-1700$ & $\begin{array}{l}\text { La gestión de mantenimiento en la empresa se fundamenta en los principios } \\
\text { básicos de la confiabilidad de activos }\end{array}$ \\
\hline
\end{tabular}

Fuente: Autores

$\mathrm{Al}$ respecto, son evidentes las necesidades de mejora en cuanto a la definición de contextos operacionales, definición de funciones primarias y secundarias y la definición de estándares de desempeño. De igual forma, en procedimientos inherentes a la evaluación de las frecuencias de fallas funcionales, la definición de los efectos de falla, así como la clasificación (de acuerdo con la norma) de las consecuencias de fallas, y el manejo de tareas de mantenimiento en función de los patrones de falla identificados.

Ahora bien, para lo anterior, se establece una metodología consistente en el cumplimiento de fases consecutivas, dentro del marco de aplicación de las herramientas del MCC, que permitirán la incorporación de aspectos inherentes a la confiabilidad de los activos, en la gestión actual de mantenimiento.

Fase 1. Definición técnica y funcional de los activos sujetos a mantenimiento. Aquí se contempla lo relacionado con el inventario, la codificación, el registro técnico, definición de estándares de desempeño, contextos operacionales e identificación de los límites de todos los activos de la empresa, así como la jerarquización de los mismos en el contexto operativo, y la determinación de funciones primarias y secundarias; asimismo, se propone en la figura 1 un sistema que permitirá dar cumplimiento con lo requerido en la norma SAE JA1012 en estos aspectos.

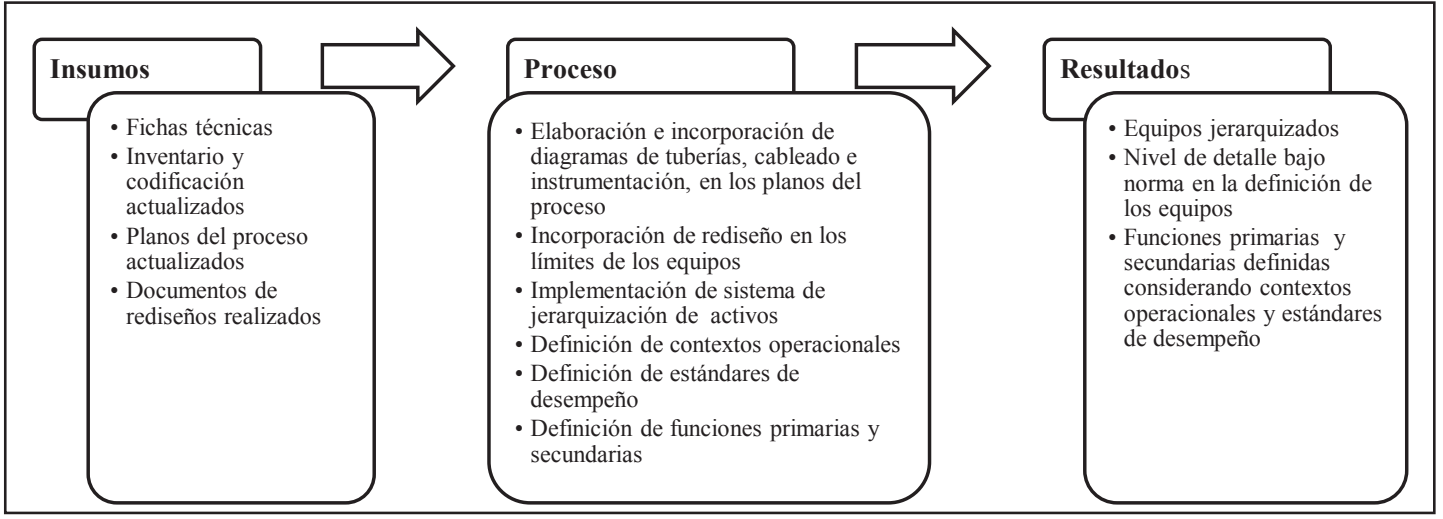

Figura 1. Sistema propuesto para la fase 1 del marco de aplicación de las herramientas del MCC Fuente: Autores 
En este sentido, la información presentada muestra la necesidad de definir estrategias orientadas a la jerarquización de equipos, que pudiera llevarse a cabo a través de la herramienta de análisis de criticidad; y estrategias orientadas hacia la definición de las funciones de equipos, que es el punto de partida de la herramienta de análisis de modos $\mathrm{y}$ efectos de fallas.
Fase 2. Definición de los modos, efectos y consecuencias de fallas funcionales de los activos sujetos a mantenimiento. Mostrada en la figura 2, comprende lo relacionado con la definición y categorización de los estados de falla, la identificación de los modos de falla, definición de los efectos y consecuencias de falla, y con el manejo de las tareas de mantenimiento en función de la categorización de las fallas.

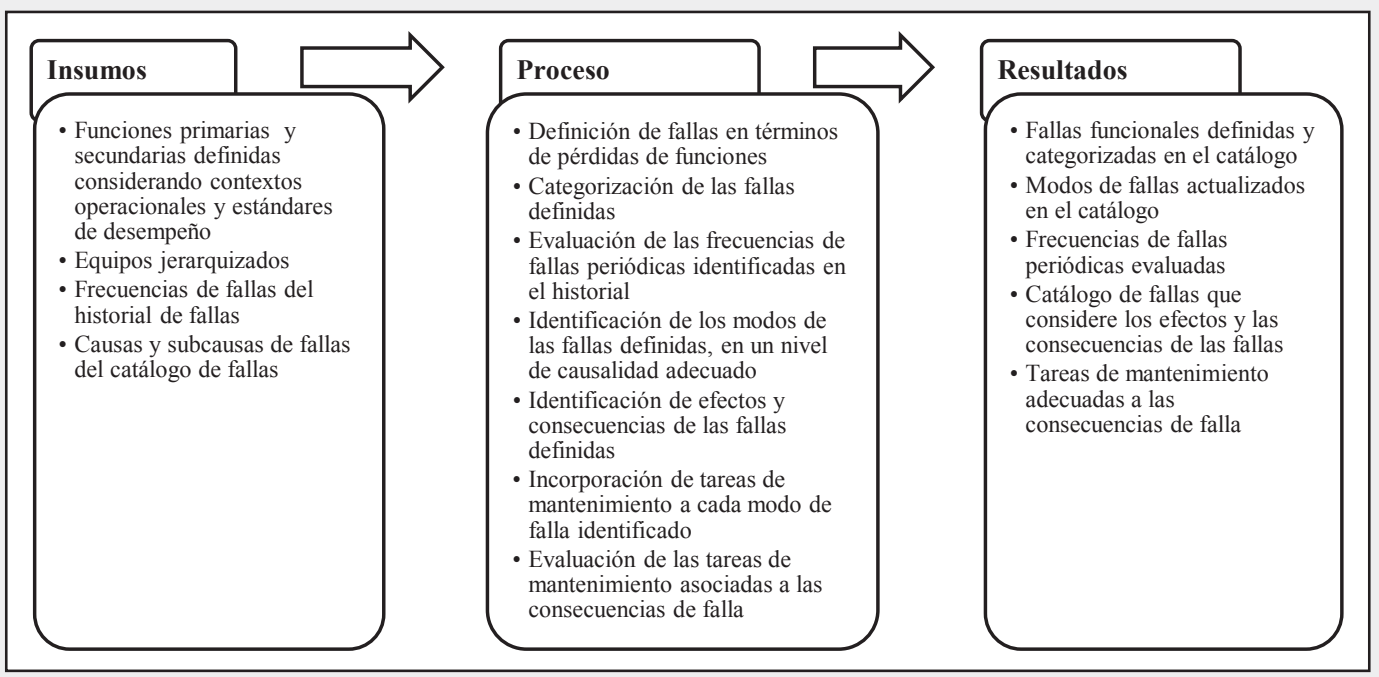

Figura 2. Sistema propuesto para la fase 2 del marco de aplicación de las herramientas del Mantenimiento Centrado en Confiabilidad

Fuente: Autores

Se puede apreciar que el proceso planteado en la segunda fase, sugiere la implantación de estrategias orientadas hacia la caracterización de las fallas, en este sentido, se requiere de la aplicación de una herramienta que permita la definición de fallas funcionales, modos, efectos y consecuencias. Además, para el proceso de la definición de modos de fallas a un nivel de causalidad adecuado (de entre 3 y 5 de acuerdo con la criticidad del equipo), se requiere la aplicación de la herramienta de análisis de causa raíz. Por otra parte, para la evaluación de las frecuencias de fallas periódicas identificadas en el historial, así como para la evaluación de las tareas de mantenimiento asociadas a cada modo de falla, se sugiere la implementación de estrategias enmarcadas en el análisis costo riesgo - beneficio; que permitan establecer la frecuencia óptima de intervención de los equipos, en función del menor impacto sobre la producción y en consideración de los costos asociados a realizar o no las actividades de mantenimiento en el tiempo especificado, asumiendo los riesgos inherentes. También, para el proceso de incorporación de tareas de mantenimiento a cada modo de falla identificado, se sugieren estrategias enfocadas al mantenimiento basado en condición, y a la optimización integral del mantenimiento, que sugieren la revisión de los objetivos estratégicos y las políticas de mantenimiento en la empresa.

Fase 3. Definición, evaluación y selección de políticas de manejo de fallas funcionales. Esta fase especificada en la figura 3, requiere de los resultados obtenidos en las fases
Enero - Junio 2016 ISSN 0122-820X E-ISSN 2422-5053 
Aplicación de herramientas de clase mundial para la gestión de mantenimiento en empresas cementeras basado en la metodología MCC

anteriores, que se convertirán en los insumos necesarios para los procesos orientados hacia la definición de políticas, y los criterios para la selección de las mismas. En este sentido, se evidencia la necesidad de utilizar herramientas gerenciales, de análisis económico, que permitan tomar decisiones con base en la mejor relación costo - beneficio para el negocio.

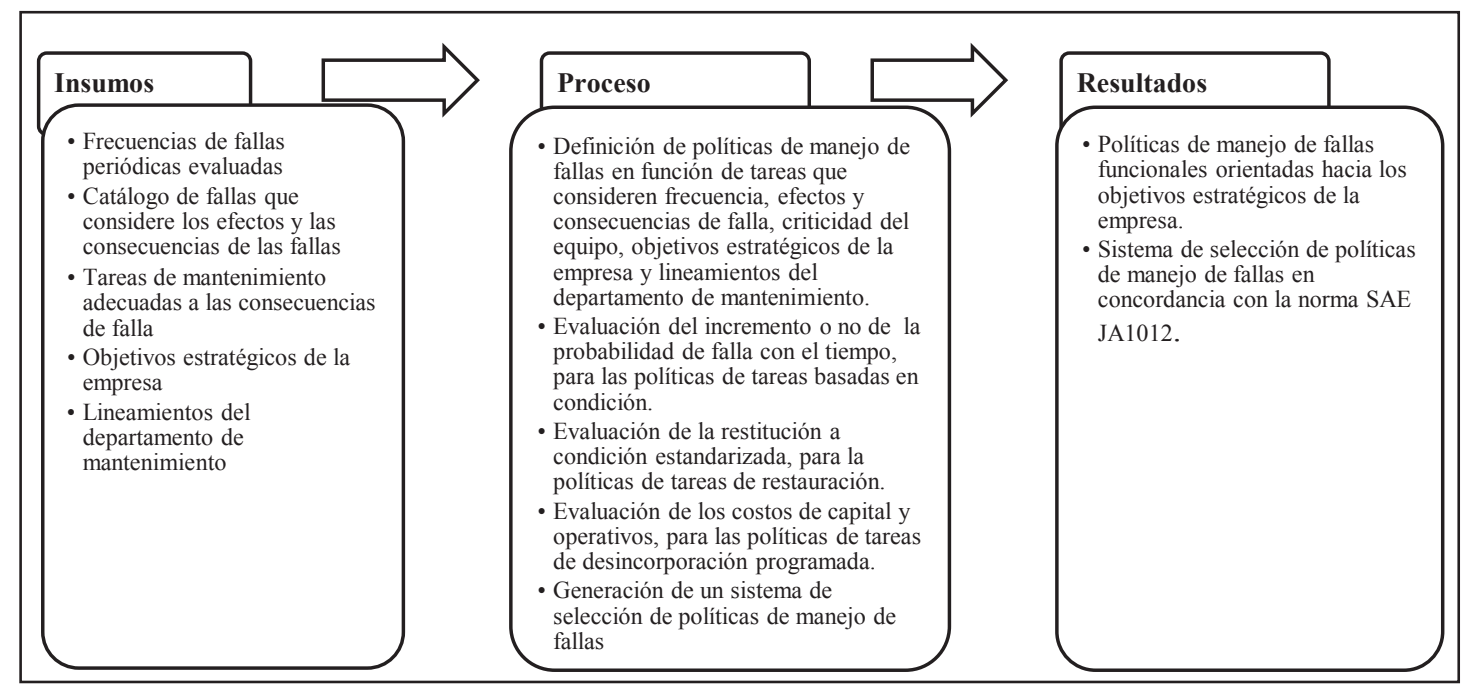

Figura 3. Sistema propuesto para la fase 3 del marco de aplicación de las herramientas del Mantenimiento Centrado en Confiabilidad Fuente: Autores

Asimismo, las herramientas de confiabilidad: análisis costo - riesgo - beneficio, y análisis del costo del ciclo de vida, pudieran permitir en el caso de la primera, una combinación óptima entre los costos de realizar una actividad y los beneficios generados, considerando el valor del riesgo que involucra la realización, o no, de tal acción; y en el caso de la segunda, elegir entre opciones de inversión o acciones de mejora, con base en el efecto sobre costo total del ciclo de vida de un activo. En línea con lo anterior, los análisis económicos permitirán establecer las políticas de restauración y/o desincorporación programada, para determinadas fallas, así como la decisión de operar hasta fallar, y del cambio de especificaciones, cuando el patrón de falla y la criticidad del equipo lo permitan, siempre considerando la relación costo - beneficio. Por otra parte, la generación de un sistema que permita seleccionar las políticas de manejo de fallas, estandarizará el proceso en la empresa, permitiendo además cumplir con lo requerido en la norma SAE JA1012.

Luego de que son identificadas las herramientas de confiabilidad que, la Fábrica Nacional de Cemento Planta Táchira, debe implementar de acuerdo con los resultados del diagnóstico, se presenta a continuación las pautas a seguir para la formulación, implementación y control de las mismas.

\section{Herramienta de Análisis de criticidad:} cuyo objetivo estratégico fue identificar el impacto de las fallas de los equipos sobre la producción y los procesos, ambiente, personas e instalaciones (ver figuras 4 y 5). 


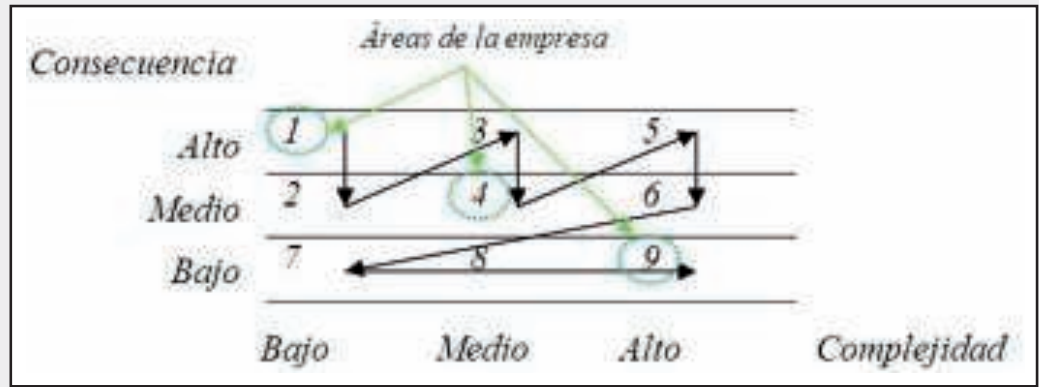

No. 1

Enero - Junio 2016

ISSN $0122-820 \mathrm{X}$

E-ISSN 2422-5053

PP: 77-88

Figura 4. Matriz de aplicación de los métodos para la medición del análisis de criticidad. 4.1 Matriz consecuencia complejidad.

Fuente: Autores

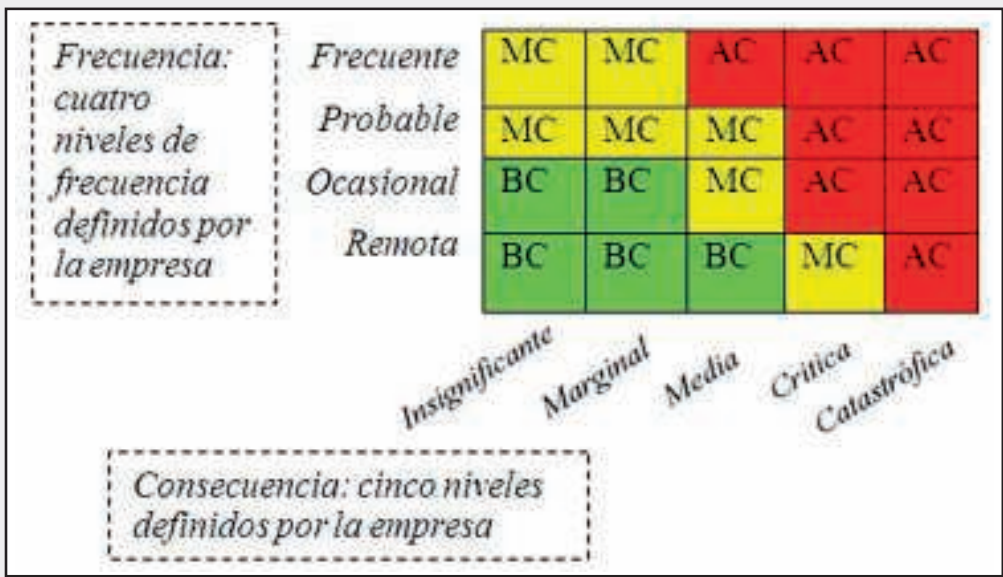

Figura 5. Matriz de aplicación de los métodos para la medición del análisis de criticidad: Matriz de consecuencia frecuencia

Fuente: Autores

Para asistir la toma de decisiones en cuanto a la economía de los recursos naturales, al servicio de la competitividad, las técnicas de control ambiental, el ofrecimiento de la mejor calidad al mejor costo, y para el manejo eficiente de los recursos asignados tomando conciencia de las relaciones costo - beneficio (ver figura 6).

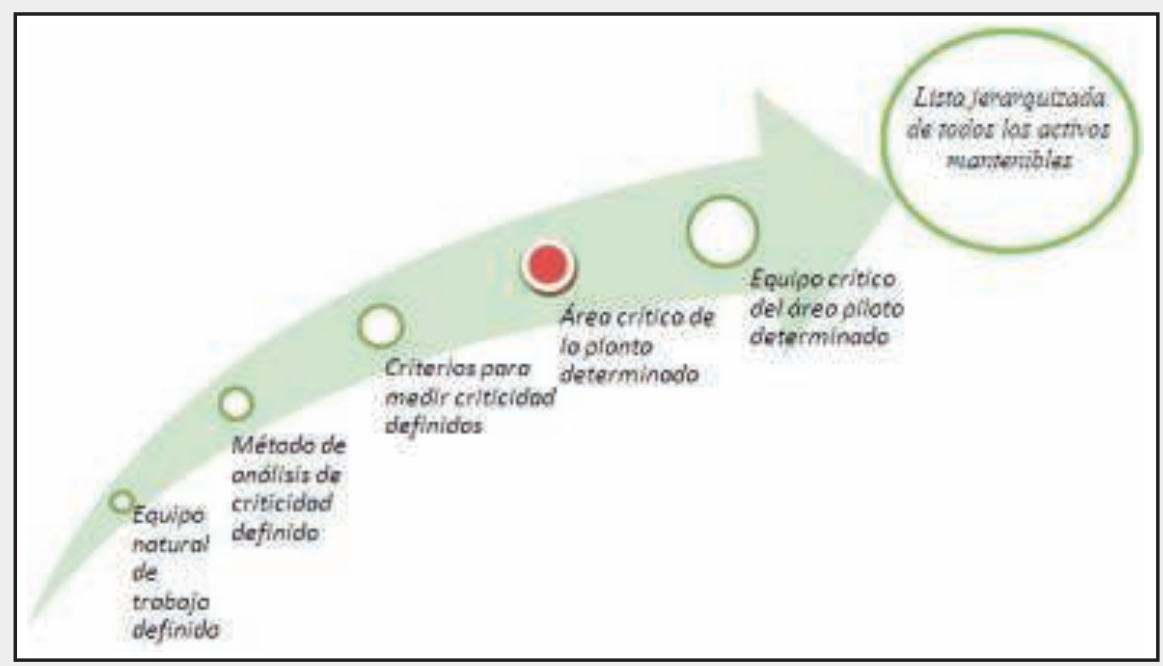

Figura 6. Ruta de implementación propuesta para la herramienta de análisis de criticidad Fuente: Autores 
No. 1

Enero - Junio 2016 ISSN 0122-820X

E-ISSN 2422-5053

En este sentido, las acciones seguidas para la implementación del análisis se resumen en la tabla III.

Tabla III. Acciones estratégicas, recursos materiales, equipo natural de trabajo e indicadores de la herramienta de análisis de criticidad definida para la Fábrica Nacional de Cemento Planta Táchira

\begin{tabular}{|c|c|c|c|}
\hline Acciones & Recursos & Equipo de trabajo & Indicadores \\
\hline $\begin{array}{l}\text { 1. Validación de los } \\
\text { métodos: conse- } \\
\text { cuencia \& comple- } \\
\text { jidad para la jerar- } \\
\text { quización de áreas, } \\
\text { y de frecuencia por }\end{array}$ & \multirow{5}{*}{$\begin{array}{l}\text { - Información técnica de } \\
\text { los equipos, planos de } \\
\text { proceso, costos de ope- } \\
\text { raciones, registro de } \\
\text { inspecciones, historial } \\
\text { de fallas. } \\
\text { - Fichas técnicas de } \\
\text { los equipos sujetos a } \\
\text { mantenimiento en el } \\
\text { Sistema Centralizado } \\
\text { de Mantenimiento } \\
\text { - Documentación de las } \\
\text { matrices de la figura } \\
\text { 4, para la aplicación de } \\
\text { los métodos. } \\
\text { Tiempo máximo de un } \\
\text { mes para la implemen- } \\
\text { tación de la estrategia }\end{array}$} & \multirow{5}{*}{$\begin{array}{l}\text { - El Jefe de Mante- } \\
\text { nimiento, Jefe de } \\
\text { Producción, Jefe de } \\
\text { Seguridad Industrial, } \\
\text { Jefe de Calidad, Jefe } \\
\text { de la Unidad Técnica } \\
\text { de Mantenimiento y } \\
\text { el Analista de Mante- }\end{array}$} & \multirow[t]{5}{*}{$\begin{array}{l}\text { - Se propuso una ruta de implementación, la cual visualmente represen- } \\
\text { ta el avance en el cumplimiento de las acciones estratégicas. Este indi- } \\
\text { cador, se esquematiza en la figura 5, el cual es valorado por el equipo } \\
\text { natural de trabajo hasta que sea de completo cumplimiento para el área } \\
\text { crítica, y luego ser expandido a las otras áreas de la Fábrica. } \\
\text { - También se propuso el indicador de equipos jerarquizados (EJE) }\end{array}$} \\
\hline $\begin{array}{l}\text { la jerarquización de } \\
\text { los equipos }\end{array}$ & & & \\
\hline $\begin{array}{l}\text { 2. Definición de } \\
\text { criterios para la } \\
\text { medición de la } \\
\text { consecuencia, com- } \\
\text { plejidad y frecuen- } \\
\text { cia de falla. }\end{array}$ & & & \\
\hline $\begin{array}{l}\text { 3. Aplicación de los } \\
\text { métodos y obten- } \\
\text { ción de la lista jerar- } \\
\text { quizada }\end{array}$ & & & \\
\hline $\begin{array}{l}\text { 4. Incorporación de } \\
\text { la criticidad en la } \\
\text { ficha técnica de los } \\
\text { equipos }\end{array}$ & & & \\
\hline
\end{tabular}

Herramienta de Análisis de fallas: cuyo objetivo estratégico fue orientar el trabajo al servicio del cliente anticipando las necesidades de mantenimiento, a través del manejo de los estados de falla funcional de los equipos sujetos a mantenimiento (ver figura 7 y 8 ).

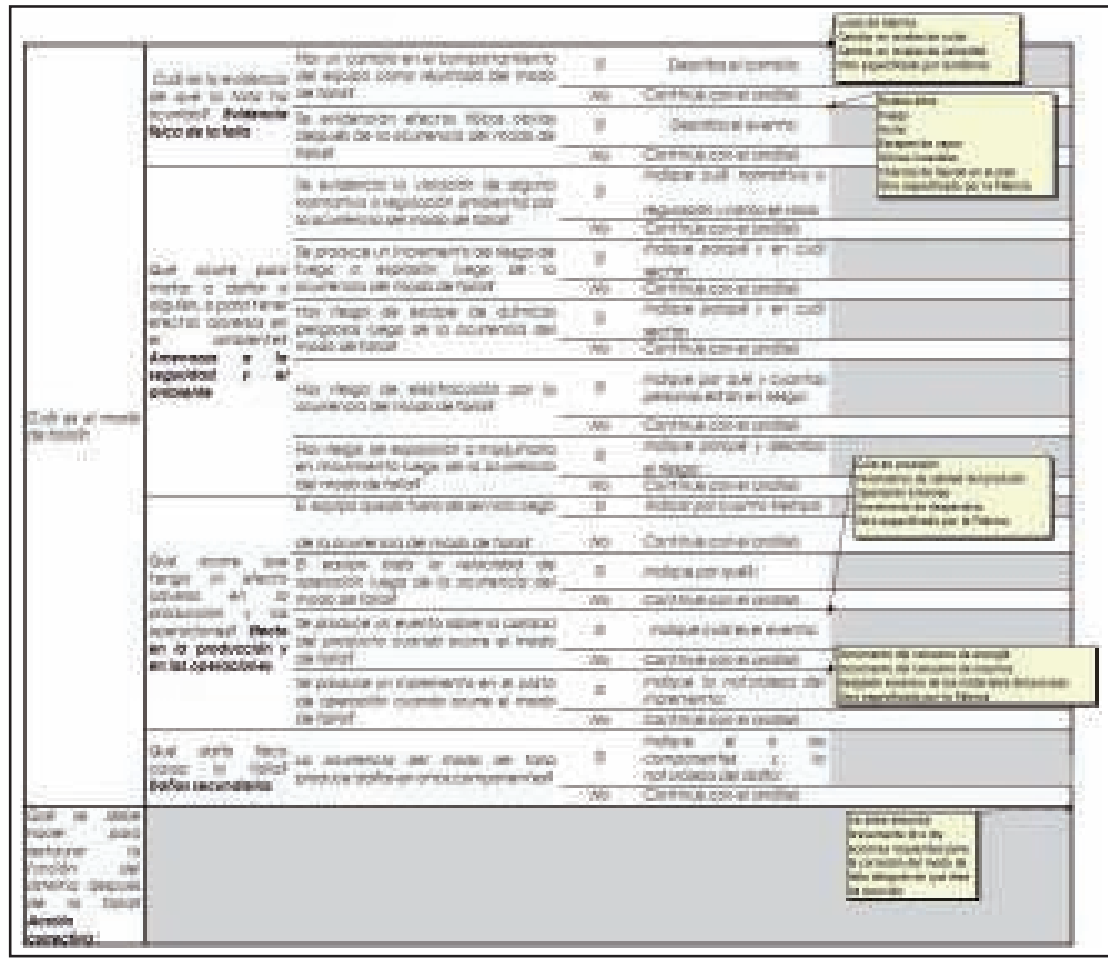

Figura 7. Formato para la documentación de los efectos de fallas Fuente: Autores 


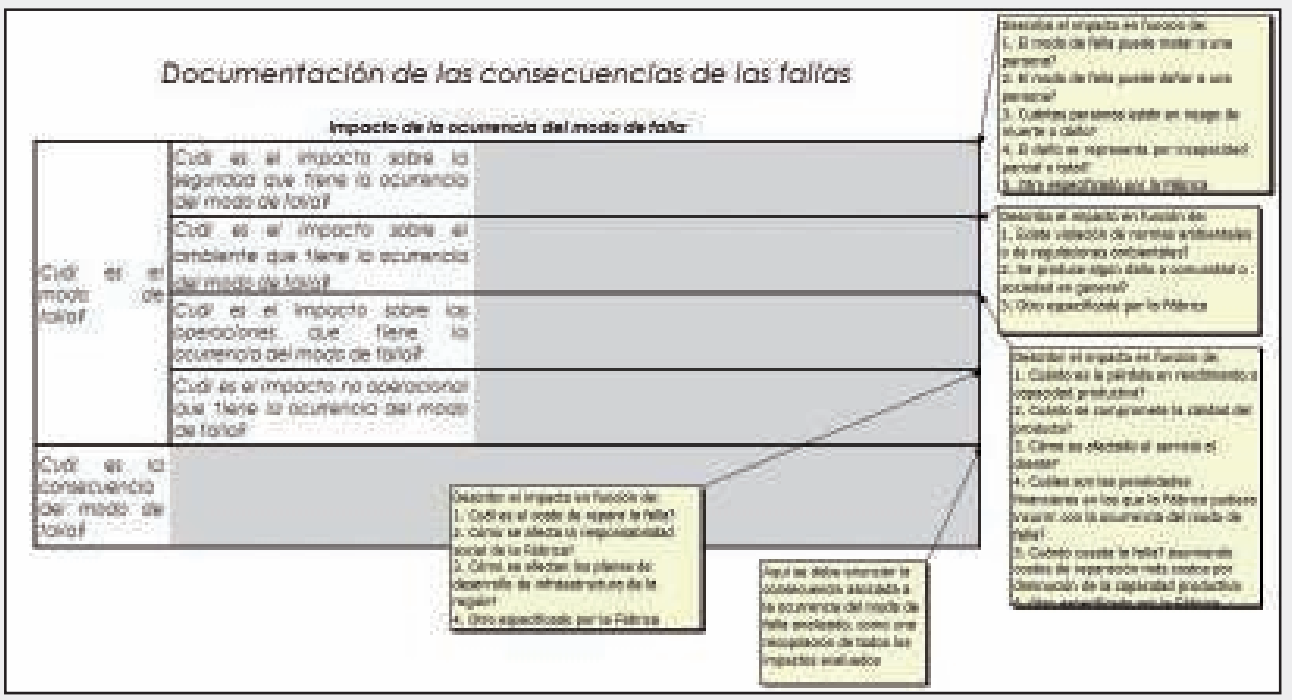

Enero - Junio 2016

ISSN 0122-820X

E-ISSN 2422-5053

PP: 77-88

Figura 8. Formato para la documentación de las consecuencias de fallas Fuente: Autores

Así mismo, las acciones seguidas para la implementación del análisis se resumen en la tabla IV.

Tabla IV. Acciones estratégicas, recursos materiales, equipo natural de trabajo e indicadores de la herramienta de análisis de fallas definida para la Fábrica Nacional de Cemento Planta Táchira

\begin{tabular}{|c|c|c|c|}
\hline Acciones & Recursos & Equipo de trabajo & Indicadores \\
\hline $\begin{array}{l}\text { 1. Definición de los estados } \\
\text { de falla asociados a la } \\
\text { función, de los equipos } \\
\text { sujetos a mantenimiento } \\
\text { 2. Clasificación de los } \\
\text { estados de falla en } \\
\text { evidentes, ocultos, totales } \\
\text { o parciales. } \\
\text { 3. Identificación de los } \\
\text { modos probables de las } \\
\text { fallas definidas, como } \\
\text { eventos por deterioros } \\
\text { físicos, diseño de equipo o } \\
\text { configuración del sistema } \\
\text { y errores humanos. }\end{array}$ & $\begin{array}{l}\text { - Hojas de trabajo para la } \\
\text { documentación de los } \\
\text { efectos y consecuencias } \\
\text { de falla, } \\
\text { - Acceso a centros de } \\
\text { costos, informes de } \\
\text { reparaciones de fallas, } \\
\text { notificaciones de } \\
\text { modos o efectos de } \\
\text { falla, informes de } \\
\text { supervisiones, histórico } \\
\text { de fallas y reportes de } \\
\text { procedimientos de } \\
\text { ejecución e de } \\
\text { instrucciones técnicas } \\
\text { Tiempo máximo de } \\
\text { cuatro meses para la } \\
\text { implementación de la } \\
\text { estrategia }\end{array}$ & $\begin{array}{l}\text { - Jefe de planta para la } \\
\text { validación de las } \\
\text { consecuencias de } \\
\text { fallas } \\
\text { El Jefe de } \\
\text { Mantenimiento, Jefe } \\
\text { de Producción, Jefe } \\
\text { de Seguridad } \\
\text { Industrial, Jefe de } \\
\text { Calidad, Jefe de la } \\
\text { Unidad Técnica de } \\
\text { Mantenimiento y el } \\
\text { Analista de } \\
\text { Mantenimiento. } \\
\text { - Jefe de } \\
\text { mantenimiento } \\
\text { eléctrico, inspector } \\
\text { eléctrico y mecánico. }\end{array}$ & $\begin{array}{l}\text { - Se propuso una ruta de implementación, la cual se } \\
\text { esquematiza en la figura } 8 \text {, y es valorada hasta que sea de } \\
\text { completo cumplimiento para el área crítica, para luego ser } \\
\text { expandida a las otras áreas de la Fábrica. Igualmente se } \\
\text { considera la realización del análisis de causa - raíz. } \\
\text { - Como indicador de seguimiento, se propuso: equipos } \\
\text { con AMEF completo (EAC). El cual será medido a partir } \\
\text { del momento de la implementación de la estrategia para el } \\
\text { equipo crítico en el área crítica. } \\
\qquad E A C=\frac{N o \text { de equipos con AMEF completado }}{N^{\circ} \text { de equipos sujetos a mantenimiento }}\end{array}$ \\
\hline $\begin{array}{l}\text { 4. Registro de la evidencia } \\
\text { física de la ocurrencia de } \\
\text { un modo de falla a través } \\
\text { del llenado del formato de } \\
\text { la figura } 7 \text {. } \\
\text { 5. Evaluación de los } \\
\text { impactos de la ocurrencia } \\
\text { de los modos de falla a } \\
\text { través de la evaluación de } \\
\text { las consecuencias en el } \\
\text { formato de la figura } 8 \text {. }\end{array}$ & & & \\
\hline
\end{tabular}


No. 1

Enero - Junio 2016 ISSN 0122-820X E-ISSN 2422-5053
La ruta de implementación propuesta para la herramienta de análisis de fallas se puede observar en la figura 9.

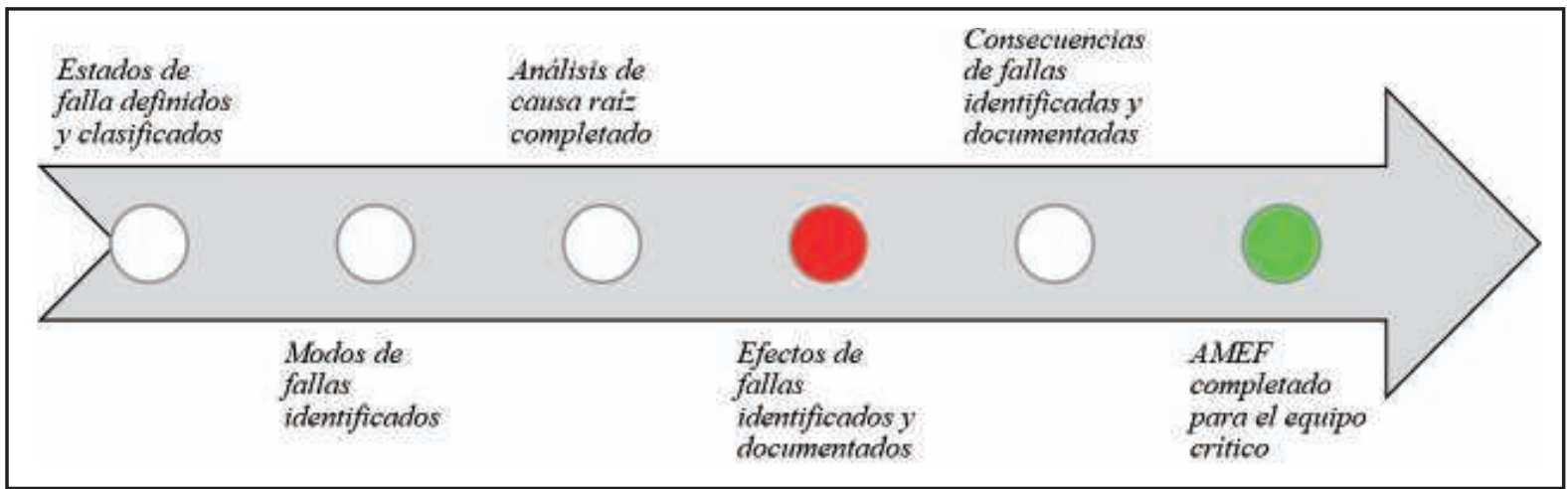

Figura 9. Ruta de implementación propuesta para la herramienta de análisis de fallas Fuente: Autores
Herramienta del análisis de causa - raíz: cuyo objetivo estratégico estuvo orientado a determinar la causa raíz de las fallas vitales de los equipos sujetos a mantenimiento, responsables de gran parte de los costos del departamento de mantenimiento, para la definición de políticas. Asimismo, las acciones seguidas para la implementación del análisis se resumen en la tabla $\mathrm{V}$.

Tabla V. Acciones estratégicas, recursos materiales, equipo natural de trabajo e indicadores de la herramienta de análisis de causa raíz definida para la Fábrica Nacional de Cemento Planta Táchira

\begin{tabular}{|c|c|c|c|}
\hline Acciones & Recursos & Equipo de trabajo & Indicadores \\
\hline $\begin{array}{l}\text { 1. Identificación de los eventos } \\
\text { y las fallas más importantes, } \\
\text { a través de la aplicación de } \\
\text { un análisis de pareto } \\
\text { mostrado en la figura } 10 . \\
\text { 2. Recaudo de las evidencias } \\
\text { de las fallas, a través de la } \\
\text { aplicación de la metodología } \\
\text { de las 5p (recoger partes, } \\
\text { posiciones, personal, papel y } \\
\text { paradigmas) } \\
\text { 3. Conformación del equipo de } \\
\text { trabajo incluyendo un } \\
\text { especialista en la aplicación } \\
\text { de la herramienta. } \\
\text { Ordenamiento del análisis } \\
\text { ACR. } \\
\text { 4. Construcción del árbol } \\
\text { lógico de las fallas vitales } \\
\text { como el que se muestra en la } \\
\text { figura 11. }\end{array}$ & $\begin{array}{l}\text { - Hojas de trabajo para } \\
\text { la elaboración de los } \\
\text { diagramas de Pareto } \\
\text { y construcción de } \\
\text { árbol lógico de fallas } \\
\text { - Acceso a centros de } \\
\text { costos, informes de } \\
\text { reparaciones de } \\
\text { fallas, notificaciones } \\
\text { de modos o efectos } \\
\text { de falla, informes de } \\
\text { supervisiones, } \\
\text { histórico de fallas y } \\
\text { reportes } \\
\text { procedimientos de } \\
\text { ejecución } \\
\text { instrucciones técnicas } \\
\text { Tiempo máximo de } \\
\text { dos meses para la } \\
\text { implementación de la } \\
\text { estrategia }\end{array}$ & $\begin{array}{l}\text { - Jefe de planta para la } \\
\text { validación de las } \\
\text { consecuencias de } \\
\text { fallas } \\
\text { - El Jefe de } \\
\text { Mantenimiento, Jefe } \\
\text { de Producción, Jefe } \\
\text { de Seguridad } \\
\text { Industrial, Jefe de } \\
\text { Calidad, Jefe de la } \\
\text { Unidad Técnica de } \\
\text { Mantenimiento y el } \\
\text { Analista de } \\
\text { Mantenimiento. } \\
\text { - Jefe de } \\
\text { mantenimiento } \\
\text { eléctrico, inspector } \\
\text { eléctrico y mecánico, } \\
\text { y un especialista en } \\
\text { la aplicación de la } \\
\text { herramienta de causa } \\
\text { - raíz }\end{array}$ & $\begin{array}{l}\text { - En conjunto con la evaluación de la estrategia anterior, } \\
\text { se propuso una ruta de implementación, la cual se } \\
\text { esquematiza en la figura } 8 \text {. } \\
\text { - Como indicador de seguimiento, se propuso: fallas } \\
\text { analizadas con causa raíz (FAI). El cual será medido a } \\
\text { partir del momento de la implementación de la estrategia } \\
\text { para el equipo crítico. } \\
F A I=\frac{N o \text { de fallas de alto impacto analizadas con } A C R}{N \text { de fallas de alto impacto }}\end{array}$ \\
\hline
\end{tabular}

Fuente: Autores

Los costos asociados a las fallas se pueden observar en la figura 10, la cantidad de dinero mayor corresponde a las fallas imprevistas columna $\mathrm{C}$ y $\mathrm{G}$, el acumulado restante corresponde a los costos asociados a las fallas recurrentes en la empresa. 


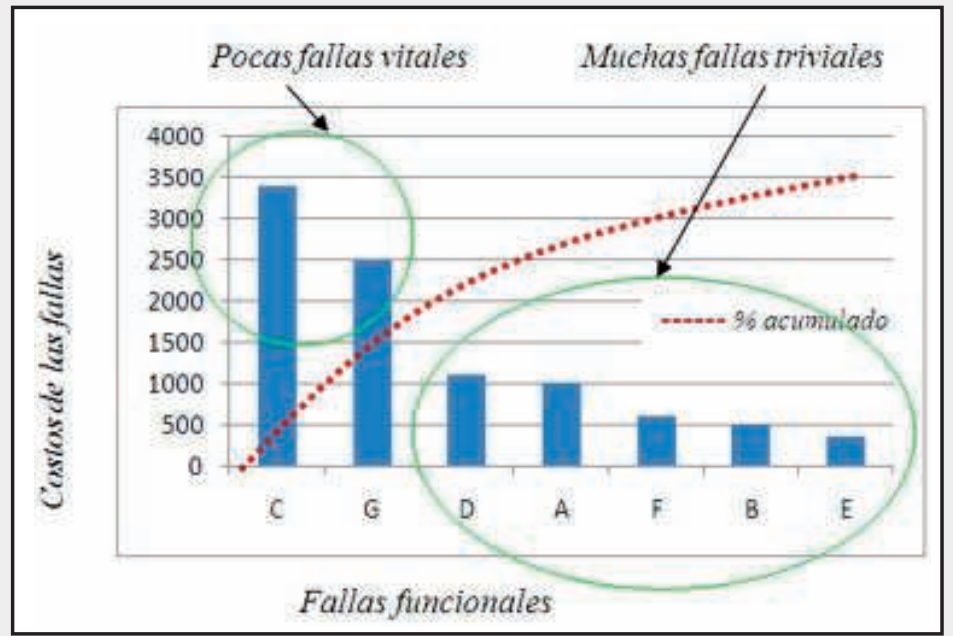

Enero - Junio 2016

Figura 10. Diagrama de Pareto donde se representan los costos de las fallas de un equipo, considerando además el porcentaje acumulado del costo total de las fallas

Fuente: Autores

En la figura 11 se presenta el árbol lógico de fallas utilizado para determinar la relación causa - efecto en las fallas presentes en las empresas cementeras estudiadas, utilizando herramientas de clase mundial.

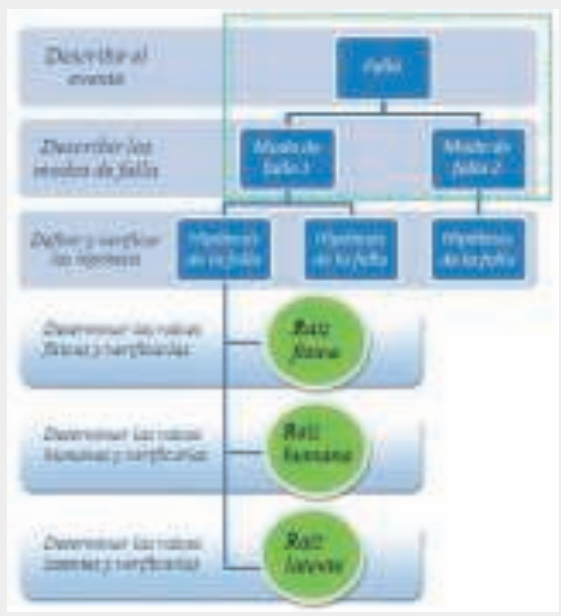

Figura 11. Esquema de Árbol lógico de fallas con niveles de causa - efecto

\section{Conclusiones}

La gestión de mantenimiento de la empresa cementera objeto de estudio, no contempla los principios básicos de la confiabilidad de los activos, puesto que presenta grandes deficiencias en aspectos básicos establecidos en la Norma SAE JA1012. Entonces, respondiendo a las interrogantes inicialmente planteadas, fue diseñado un marco referencial para la aplicación de herramientas de clase mundial, enmarcadas en la metodología del MCC, el cual contempla el cumplimiento de tres fases en la empresa, contando con los insumos indicados para cada fase. Luego, se identificaron las herramientas de análisis 
No. 1

Enero - Junio 2016 ISSN 0122-820X

E-ISSN 2422-5053 PP: 77-88 de criticidad, análisis de modos y efectos de fallas y análisis de causa - raíz, como requeridas por la gestión de mantenimiento para el cumplimiento de objetivos estratégicos. Finalmente se definió la implantación de estas herramientas, través de la formación de equipos naturales de trabajo, y el cumplimiento de acciones estratégicas, medidas por indicadores de implementación y seguimiento en cada caso.

\section{Agradecimientos:}

Los autores expresan su agradecimiento al Decanato de Postgrado de la Universidad Nacional Experimental del Táchira, a la Fábrica Nacional de Cementos Planta Táchira y a los Profesores pertenecientes al Programa de Sistematización de la Función Mantenimiento del Decanato de Investigación de la UNET.

\section{Referencias}

[1] E. Primera. "RCM en plantas cementeras. Maintenance Main - Tech Technology". Planta Pertigalete, [online]. Disponible en: http://es.scribd.com/doc/283455342/ Plantas-Cementeras-Final\#.

[2] C. Parra, R. López, A. Balda, A. Crespo. "Modelo Integral para Optimizar la Confiabilidad en Instalaciones Petroleras". Presentada en 2004 en 19 Congreso Brasilero de Mantenimiento, Curitiba, Brasil.
[3] N. B. Bloom. Reliability Centered Maintenance (RCM) Implementation Made Simple, United States of America, McGrawHill, 2006.

[4] B. S. Dhillon. Engineering Maintenance: A Modern Approach, United States of America, CRC Press LLC, 2002.

[5] E. A Fuenmayor P. "Análisis de Reemplazo de un Activo Basado en Costos de Ciclo de Vida”. Confiabilidad
Industrial. Asociación Venezolana de Profesionales de Mantenimiento y Confiabilidad. Año 3, no. 11, pp.12-14, Venezuela: GTS Confiablidad, 2011.

[6] B. A Aguinaga. "Confiabilidad Operacional para la Ingeniería del Mantenimiento". Presentado en 2007 en $8^{\text {vo }}$ Congreso Iberoamericano de Ingeniería Mecánica, Quito, Ec.

[7] Norma SAE JA1011. Criterios de Evaluación para Procesos de Mantenimiento Centrado en Confiabilidad. Norma para vehículos aeroespaciales y de superficie. ISO estándar: Ginebra, Suiza, 1.999.

[8] Norma SAE JA1012. Una Guía para la Norma del Mantenimiento Centrado en Confiabilidad (MCC). Prácticas recomendadas Para Vehículos Aeroespaciales y de Superficie. ISO estándar: Ginebra, Suiza, 2002. 